\title{
Relativistic point-form approach to hadron properties
}

\section{Willibald Plessas*}

Institute of Physics, University of Graz, Universitätsplatz, 5, A-8010 Graz, Austria

E-mail: plessas@uni-graz.at

We present a review of the description of hadron properties along an invariant mass operator in the point form of Poincaré-invariant relativistic dynamics. The quark-quark interaction is furnished by a linear confinement, consistent with the QCD string tension, and a hyperfine interaction derived from Goldstone-boson exchange. The main advantage of the point-form approach is the possibility of calculating manifestly covariant observables, since the generators of Lorentz transformations remain interaction-free. We discuss the static properties of the mass-operator eigenstates, such as the invariant mass spectra of light- and heavy-flavor baryons, the characteristics of the eigenstates in terms of their spin, flavor, and spatial dependences as well as their classification into spin-flavor multiplets. Regarding dynamical observables we address the electroweak structures of the nucleon and hyperon ground states, including their electric radii, magnetic moments as well as axial charges, and in addition a recently derived microscopic description of the $\pi N N$ as well as $\pi N \Delta$ interaction vertices. Except for hadronic resonance decays, which are not addressed here due to space limitations, all of these observables are obtained in good agreement with existing phenomenology. Relativistic (boost) effects are generally sizable. We conclude that low-energy hadrons can be well described by an effective theory with a finite number of degrees of freedom, as long as the symmetries of low-energy quantum chromodynamics (spontaneously broken chiral symmetry) as well as special relativity (Poincaré invariance) are properly taken into account. The latter requirement is particularly well and efficiently met in the point-form approach.

Light Cone 2010 - LC2010

June 14-18, 2010

Valencia, Spain

\footnotetext{
${ }^{*}$ Speaker.
} 


\section{Motivation for the relativistic constituent-quark model}

Up till now all attempts of solving quantum chromodynamics (QCD) are still limited. They are either constrained to certain energy domains (such as perturbative QCD), have to resort to truncations (such as effective field theories or functional approaches), or face severe numerical limitations (such as lattice QCD). Over the past decades we have also learnt, however, that it is not absolutely necessary to take into account all aspects of QCD in order to achieve a consistent quantitative description of hadron properties, especially in the domain of low and intermediate energies. Rather it has turned out sufficient to include into a theory the relevant/active degrees of freedom. Most reliably these degrees of freedom can be identified by considering the symmetries governing a particular system. With regard to hadrons (at low energies) such symmetries are certainly suggested by the spontaneous breaking of chiral symmetry $(\mathrm{SB} \chi \mathrm{S})$ of QCD in the low-energy domain and by special relativity, i.e. invariance under Poincare transformations. In other words, the dynamics we employ for the description of hadrons has to fulfill (at least) the constraints following from these invariance conditions.

From this point of view, we may formulate the relativistic constituent-quark model (RCQM). It describes hadrons as few-quark systems along a relativistically invariant, interacting mass operator $\hat{M}=\hat{M}_{\text {free }}+\hat{M}_{\text {int }}$. In this way, we adhere to a Hamiltonian theory restricted to a finite number of degrees of freedom and not a field theory. As a consequence it is certainly limited to a restricted (energy) domain. As soon as further degrees of freedom appear beyond the ones initially assumed, the theory is no longer adequate and has to be extended to include them too; this can be done in principle but in the end requires considerably more computational efforts. The principal advantages of such a Hamiltonian approach are that

- the theoretical framework is rigorously established, as a Poincaré-invariant quantum theory formulated on a given Hilbert space, and

- the corresponding dynamical equations (specifically the eigenvalue problem of the mass operator) can be solved to any desired accuracy, as long as a reasonable finite number of degrees of freedom are involved.

In this spirit, we view hadrons - and possibly other objects allowed by QCD - as consisting of a finite number of constituents interacting by dynamics that strictly conform to the symmetry constraints from the observed/given invariances.

\section{The Goldstone-boson exchange RCQM}

In this contribution we are essentially concerned with baryon properties at low and intermediate energies. In this context we use primarily the RCQM that assumes baryons to consist of three confined constituent quarks whose dynamics is mediated by Goldstone-boson exchange (GBE); the corresponding predictions will be compared also with other modern RCQMs. The main idea of the GBE RCQM is that the constituents as well as the exchange bosons are generated by the $\mathrm{SB} \chi \mathrm{S}$ of $\mathrm{QCD}$, which means, specifically in the three-flavor case, the breaking of $S U(3)_{L} \times S U(3)_{R} \rightarrow S U(3)_{V}$. Consequently, we have constituent quarks as quasiparticles with a dynamical mass and Goldstone bosons as the generators of the $S U(3)_{V}$ transformations. The latter 
give rise to the forces between the confined constituent quarks and materialize themselves through pseudoscalar mesons. The effective interaction Lagrangian is thus given by

$$
\mathscr{L}_{\text {int }} \sim i g \bar{\psi} \gamma_{5} \vec{\lambda} \cdot \vec{\phi} \psi
$$

where $\psi$ are the fermion (constituent quark) fields coupled by the (Goldstone) boson fields $\vec{\phi}$ through a pseudoscalar-type coupling involving the $S U(3)$ flavor matrices $\vec{\lambda}^{F}$ with a strength $g$.

After such type of dynamics had been suggested by Glozman and Riska for baryons at low energies [1], the Graz group constructed a first nonrelativistic constituent quark model whose hyperfine interaction was deduced from the interaction in eq. (2.1) and led to a quark-quark potential with explicit flavor dependence [2]. Soon it was realized that a nonrelativistic quark model is by no means adequate and one has to work in a relativistic framework. This resulted in the so-called pseudoscalar Goldstone-boson exchange relativistic constituent quark model (psGBE RCQM) [3]. It initially relied only on the spin-spin part of the GBE interaction but was later on extended to include in addition all central, tensor, and spin-orbit interactions (EGBE RCQM) [4]. For the details of the GBE RCQMs we refer to the original papers.

\section{Baryon spectroscopy}

The remarkable features of the GBE dynamics for constituent quarks are already seen in the baryon excitation spectra, where for the first time the right level orderings of positive- and negativeparity resonances could be reproduced simultaneously in the $N$ and $\Lambda$ spectra [5]. Obviously this achievement is due to the specific flavor dependence in the pertinent hyperfine potential. Such a behaviour is much in contrast to other recent RCQMs, like the one-gluon-echange (OGE) RCQM [6]

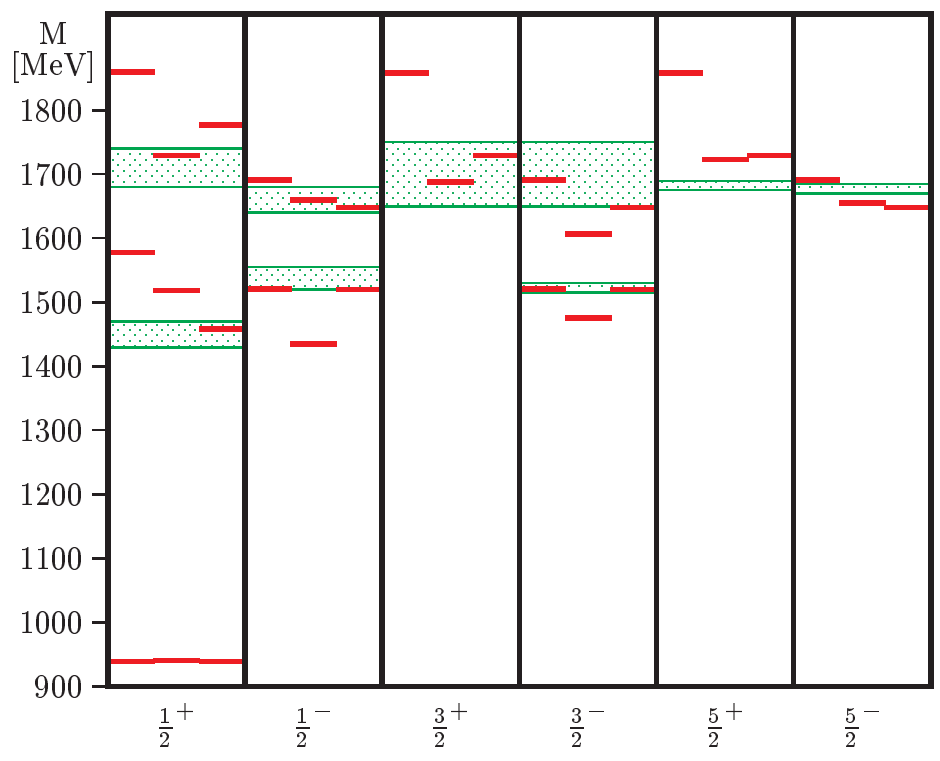

Figure 1: Nucleon excitation spectra of three different types of RCQMs. In each column of definite $J^{P}$ the left horizontal lines represent the results of the relativistic variant of the Bhaduri-Cohler-Nogami OGE RCQM [6], the middle ones of the II RCQM (Version A) [7], and the right ones of the psGBE RCQM [3]. The shadowed boxes give the experimental data with their uncertainties after the PDG [8]. 
or the one whose hyperfine potential is deduced from instanton-induced (II) interactions [7], cf. the comparisons in figs. 1 and 2; for the complete baryon excitation spectra see the original references given above.

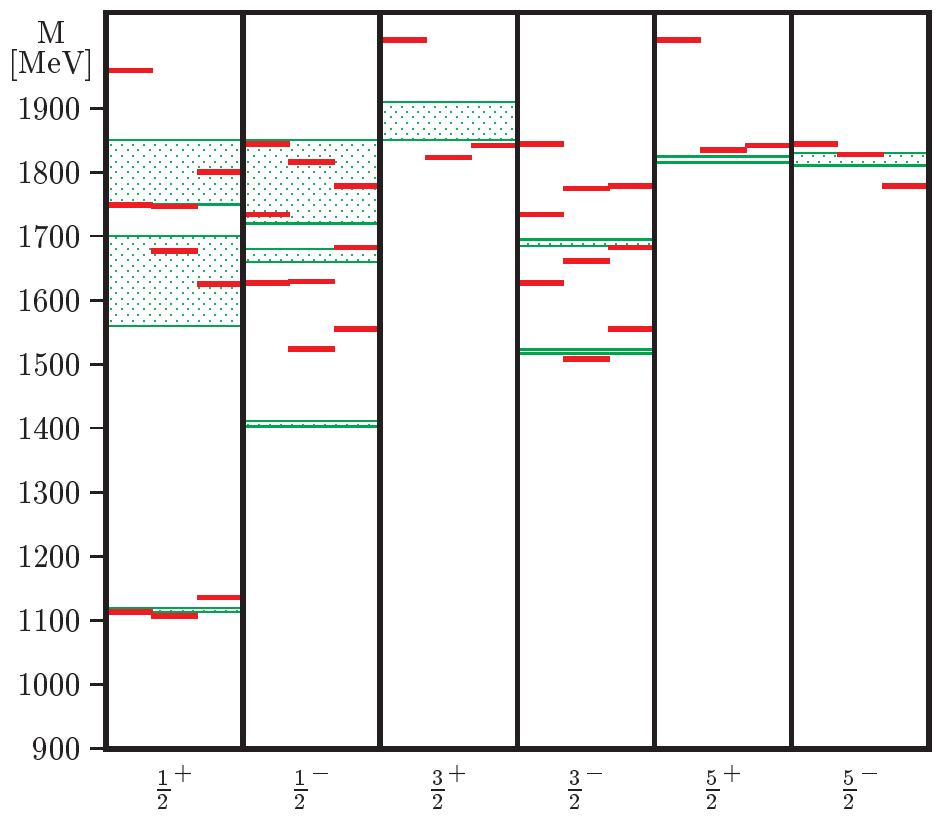

Figure 2: $\Lambda$ excitation spectra. Same caption as in fig. 1.

The action of the hyperfine forces in the GBE RCQMs is explained in detail in ref. [5]. There it is also shown, how the level orderings come about, as one steps out from the case with the confinement interaction only and gradually increases the size of the meson-quark coupling until its final strength is reached, which is the value deduced from the pion-nucleon coupling constant via the Goldberger-Treiman relation. Later on such a behaviour of the level shifts of positive- and negative-parity resonances has also been found in lattice-QCD calculations, when one approaches the chiral limit (see, e.g., ref. [9]).

From fig. 2 also a severe shortcoming of all the contemporary RCQMs that rely on three-quark configurations is evident, namely, the failure of reproducing the $\Lambda(1405)$ resonance. Obviously, for this particular case additional degrees of freedom, specifically the coupling to the $K-N$ channel, are needed. To our knowledge this has not yet been satisfactorily achieved with a relativistically invariant mass operator or in any other relativistic framework.

\section{Electroweak structures of baryon ground states}

Once we have solved the eigenvalue problem of the invariant mass operator, we are also equipped with the eigenstates of the baryon ground states and resonances. They can be further employed for the calculation of baryon reactions. In this section we consider the electromagnetic and weak form factors of the baryon ground states.

First of all it is interesting to look at the spatial probability distributions of these mass-operator eigenstates in the rest frame. Corresponding pictures can be found in ref. [10]. They exhibit 
particular structures typical for the classification of ground states and resonances into spin-flavor multiplets. On the basis of these spatial structures and the strong-decay patterns of the pertinent resonances a modified and extended classification scheme of baryons into $S U(3)_{F}$ flavor multiplets has been developed [8]. An aspect worth to be emphasized is the relatively dense probability distribution at short distances in configuration space. For instance, in case of the $N$ ground state it is maximal at magnitudes of the Jacobi coordinates of $\xi \approx \eta \approx 0.3 \mathrm{fm}$. The probability distributions for all ground states furthermore exhibit essentially spherical symmetry and do not show any pronounced quark-diquark structures [10]. Of course, with regard to certain subtle observables, e.g., the electric neutron form factor (see below), small but important mixed-symmetric spatial configurations in the wave functions are crucially important.

For the calculation of baryon reactions, here specifically the electroweak form factors as well as electric radii and magnetic moments, one has to know how to boost the eigenstates. This task can most efficiently be carried out for general Lorentz transformations in the point form of Poincaré-invariant quantum mechanics, since in this case the generators of rotations and boosts are interaction-free. Thereby one can calculate manifestly covariant observables.

The results obtained so far for electroweak observables of baryons in the point-form approach have all been calculated with electromagnetic and axial currents according to the so-called pointform spectator-model (PFSM) [11]. These approximative currents are defined by the exchange boson coupling to only one of the constituent quarks, while the momentum is transferred to the baryon as a whole. Consequently, they amount to effective many-body currents [12].

In figs. 3 and 4 we first show the electromagnetic nucleon form factors as functions of the momentum transfer $Q^{2}$. The PFSM predictions are found in surprisingly good agreement with the experimental data both for the proton and the neutron $[13,14]$. This is essentially true for both the psGBE and OGE RCQMs. In addition, the corresponding results compare well with the relativistic predictions by the II RCQM from a Bethe-Salpeter approach. Only, the calculation with the wave function from confinement only, which is spatially completely symmetric, fails drastically; e.g., it yields an almost zero result for the neutron electric form factor (cf. the right panel of fig. 3).

A nonrelativistic approach is by no means adequate for the electromagnetic form factors [13, 14]. A comparison of the point-form with the instant-form approach has revealed that in the spectator approximation for the electromagnetic current, the instant form falls short too [12] (see figs. 5 and 6). In particular, it was found that the instant-form spectator-model (IFSM) construction is not frame-independent. This represents a considerable disadvantage as compared to the PFSM, which strictly maintains its spectator-model character in all reference frames and thus leads to manifestly covariant observables.

In view of the relatively small extensions of the spatial probability distributions of the nucleon wave functions in the rest frame, being centered around $\sim 0.3 \mathrm{fm} \mathrm{[10],} \mathrm{one} \mathrm{gets} \mathrm{certainly} \mathrm{curious,}$ how the electric radius especially of the proton (of $r_{E} \sim 0.877 \mathrm{fm}$ ) can be reproduced. Here the relativistic calculations along the PFSM again yield very reasonable predictions [15] (see tab. 1). The same is true for the neutron and in case of the $\Sigma^{-}$(not quoted here), for the $r_{E}$ of which experimental data exist too. Also the magnetic moments are at once described correctly, with only minor deviations from phenomenology (see tab. 2). Again the IFSM results fail drastically like the nonrelativistic impulse approximation (NRIA) does [12].

The axial nucleon form factors are also well described in the PFSM approach $[16,14]$ (see 

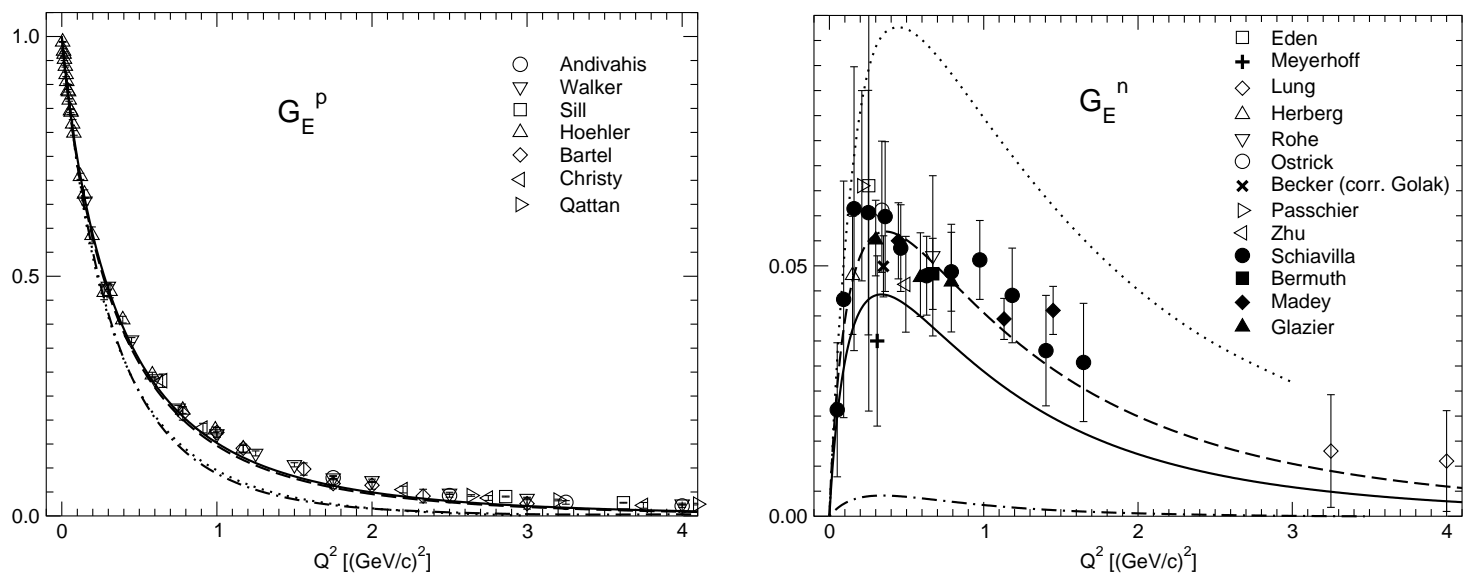

Figure 3: Predictions of the psGBE RCQM for the electric proton and neutron form factors as calculated along the PFSM (solid lines) in comparison to the results from the Bhaduri-Cohler-Nogami OGE RCQM calculated also with the PFSM (dashed lines) and the results from the II RCQM calculated in a BetheSalpeter approach (dotted lines). A comparison is also given to the case with confinement only, i.e. with a spatially symmetric wave function (dashed-dotted line). Experimental data as specified in the inserts, see also ref. [12].
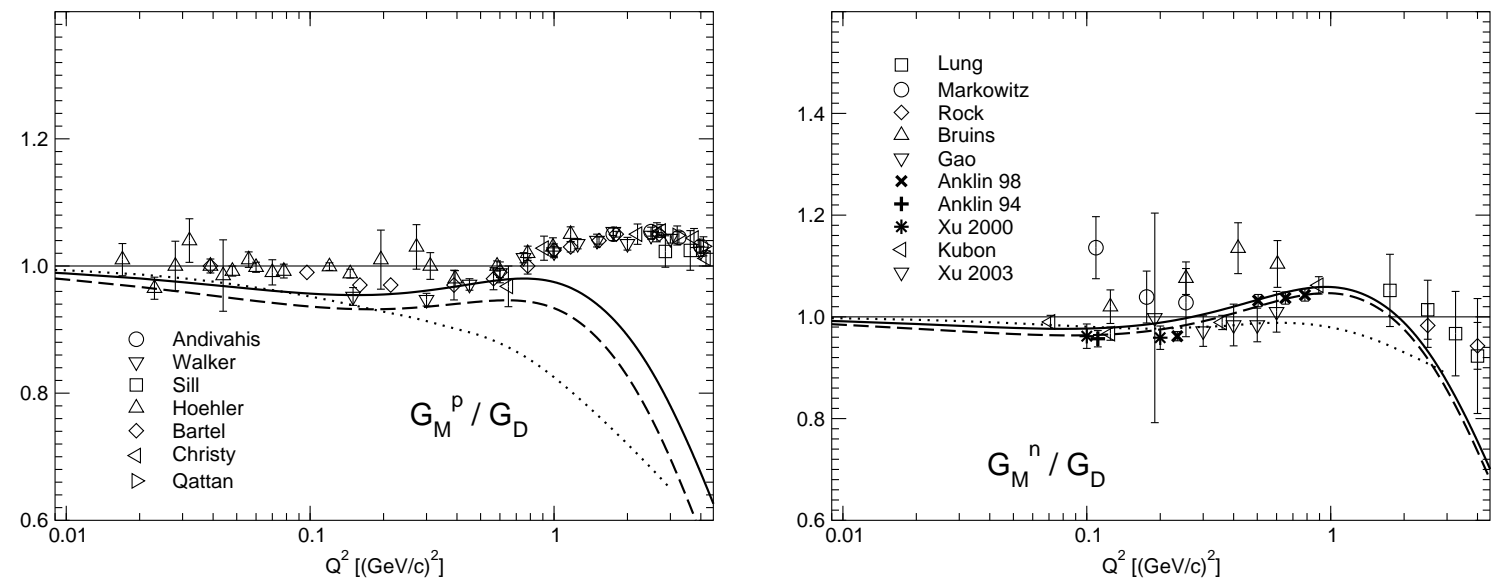

Figure 4: Same comparison as in fig. 3 but for the magnetic proton and neutron form factors as represented by ratios to the dipole form.

fig. 7). Similarly, the axial charges of the nucleon as well as other baryon ground states turn out as reasonable [17] (see tab. 3, where the predictions of the EGBE RCQM are quoted). A study of the axial charges of $N^{*}$ and other baryon resonances has in addition revealed that the predictions of the GBE RCQM agree well with data so far known from lattice QCD [17, 18].

\section{Structure of meson-baryon interaction vertices}

The baryon axial properties are closely related to the meson-baryon couplings through the Goldberger-Treiman relation. Therefore, on has recently also studied the predictions of the psGBE RCQM for the structures of the $\pi N N$ as well as $\pi N \Delta$ interaction vertices [23]. It has turned out 

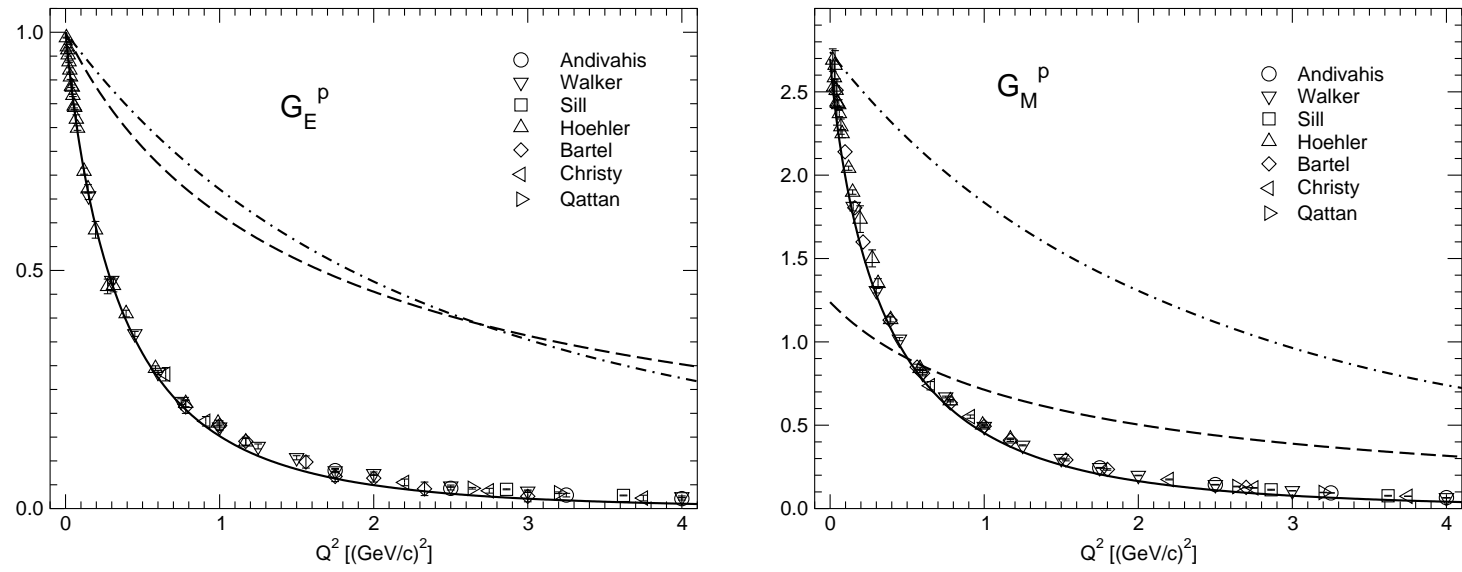

Figure 5: Comparison of the electric and magnetic form factors of the proton as calculated with the psGBE RCQM along the PFSM (full line) and the IFSM (dashed line). Also the nonrelativistic impulse approximation is given (dash-dotted line). Experimental data as specified in the inserts, see also ref. [12].
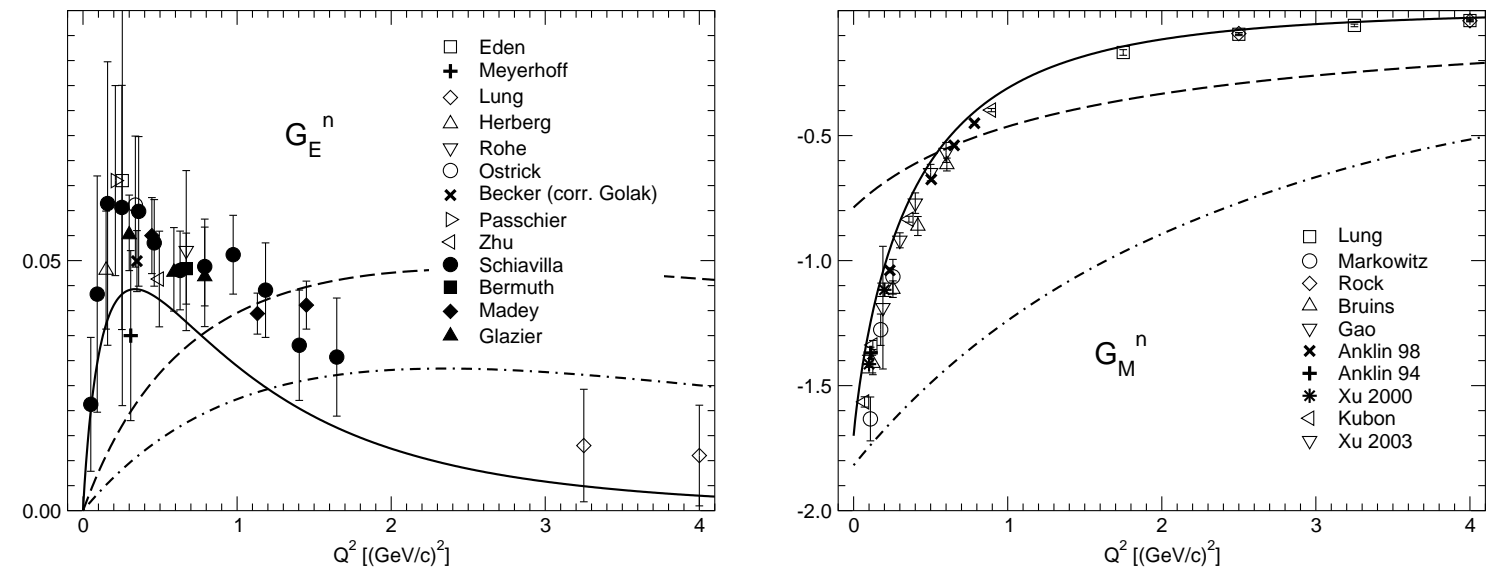

Figure 6: Same comparison as in fig. 5 but for the neutron.

that the relativistic microscopic theory relying on the meson-quark coupling provided by the GBE dynamics yields quite reasonable descriptions of the momentum dependences of these strong form factors, in the sense that previous parameterizations within phenomenological meson-nucleon or meson- $\Delta$ models $[24,25,26]$ have arrived at rather similar results (see fig. 8). Of course, the strong vertex form factors $G_{\pi N N}$ and $G_{\pi N \Delta}$ are not experimentally observable, and a comparison is possible only to other theoretical approaches. Lattice-QCD results exist from various groups but, unfortunately, they do not yield a unique picture. In view of the considerable differences of the RCQM predictions with the lattice-QCD results and of the lattice-QCD results among themselves, it would certainly be desirable to have a more reliable data base for the comparison. In any case, it should be noted that the magnitude of the $\pi N N$ coupling constant $f_{\pi N N}$ extracted from the vertex form factors of the GBE RCQM is not only in good agreement with the phenomenological value of 0.075 but also fits to the nucleon axial charge through the Goldberger-Treiman relation [23]. 


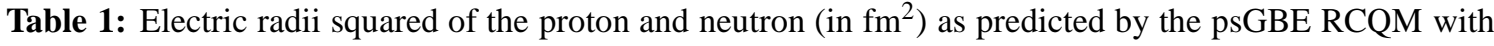
the PFSM, IFSM, and the NRIA current operators. Additional results (for all of the baryon ground states) can be found in ref. [15]. Experimental data after the PDG [8].

\begin{tabular}{ccrrr} 
Nucleon & Experiment & PFSM & IFSM & NRIA \\
\hline $\mathrm{p}$ & $0.769 \pm 0.012$ & 0.824 & 0.156 & 0.102 \\
$\mathrm{n}$ & $-0.1161 \pm 0.0022$ & -0.135 & -0.020 & -0.116 \\
\hline
\end{tabular}

Table 2: Magnetic moments of the proton and neutron (in n.m.) as predicted by the psGBE RCQM with the PFSM, IFSM, and the NRIA current operators. Additional results (for all of the baryon ground states) can be found in ref. [15]. Experimental data after the PDG [8].

\begin{tabular}{ccrrr} 
Nucleon & Experiment & PFSM & IFSM & NRIA \\
\hline $\mathrm{p}$ & $2.792847356 \pm 0.000000023$ & 2.70 & 1.24 & 2.74 \\
$\mathrm{n}$ & $-1.9130427 \pm 0.0000005$ & -1.70 & -0.79 & -1.82 \\
\hline
\end{tabular}

Table 3: Axial charges $g_{A}^{B}$ of octet and decuplet baryon ground states as predicted by the EGBE RCQM [4] in comparison to experiment [8] and lattice-QCD results from Lin and Orginos (LO) [19] and Erkol, Oka, and Takahashi (EOT) [20] as well as results from chiral perturbation theory by Jiang and Tiburzi (JT) [21, 22]; also given is the nonrelativistic limit (NR) from the EGBE RCQM.

\begin{tabular}{ccccccc} 
Baryon & Experiment & EGBE & LO & EOT & JT & NR \\
\hline$N$ & $1.2694 \pm 0.0028$ & 1.15 & $1.18 \pm 0.10$ & $1.314 \pm 0.024$ & 1.18 & 1.65 \\
$\Sigma$ & $\ldots$ & 0.65 & $0.636 \pm 0.068^{\dagger}$ & $0.686 \pm 0.021^{\dagger}$ & 0.73 & 0.93 \\
$\Xi$ & $\ldots$ & -0.21 & $-0.277 \pm 0.034$ & $-0.299 \pm 0.014^{\dagger}$ & $-0.23^{\dagger}$ & -0.32 \\
\hline$\Delta$ & $\ldots$ & -4.48 & $\ldots$ & $\ldots$ & $\sim-4.5$ & -6.00 \\
$\Sigma^{*}$ & $\ldots$ & -1.06 & $\ldots$ & $\ldots$ & $\ldots$ & -1.41 \\
$\Xi^{*}$ & $\ldots$ & -0.75 & $\ldots$ & $\ldots$ & $\ldots$ & -1.00 \\
\hline
\end{tabular}

$\dagger$ Because of another definition of $g_{A}^{\Sigma}$ this numerical value is different by a $\sqrt{2}$ from the one quoted in the original paper.

$\ddagger$ Because of another definition of $g_{A}^{\Xi}$ this value has a sign opposite to the one in the original paper.

\section{Concluding remarks}

The point form has not been much used until about a decade ago. Once first results on elastic $N$ form factors have become available around the year 2000, they appeared as rather surprising and could be seen as incidentally in agreement with phenomenology. However, with the advent of ever more predictions derived along the point form, this approach has become truly remarkable, especially since the very reasons for the good performance are not yet fully understood. Further studies are thus strongly advised, above all consistent comparisons with the front and instant forms. 

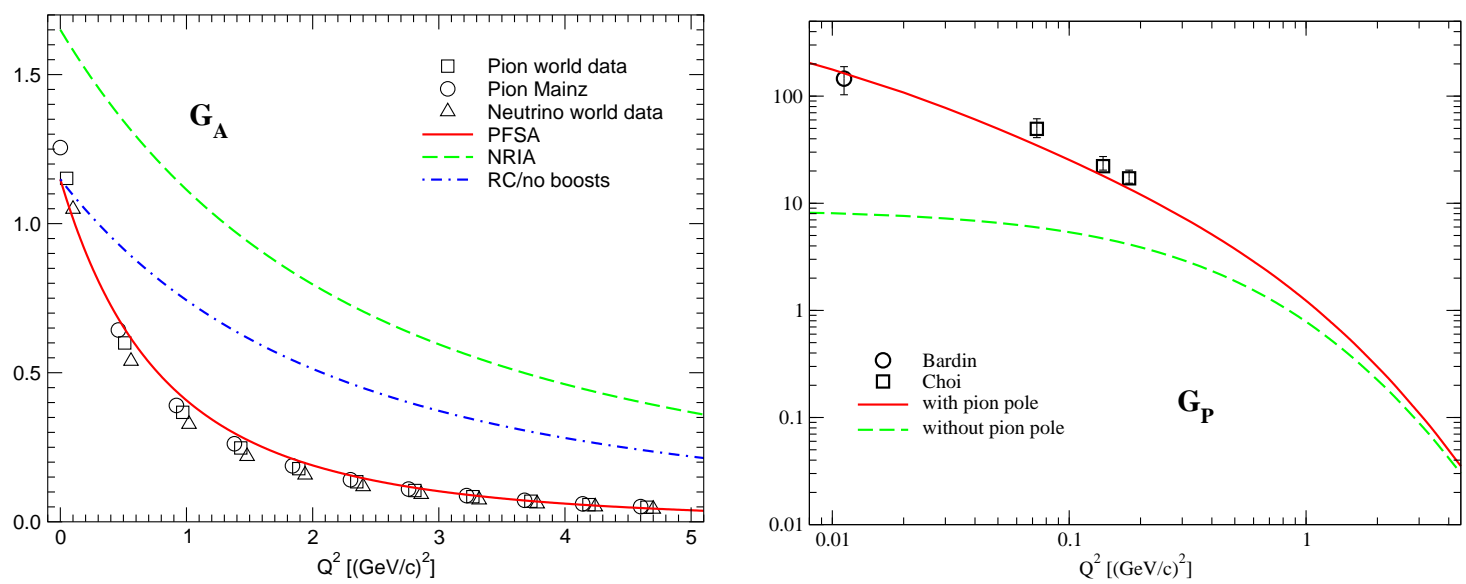

Figure 7: Predictions of the psGBE RCQM for the axial (left) and induced pseudoscalar form factors (right) of the nucleon as calculated along the PFSM (solid/red lines). A comparison is given to the cases with a relativistic current but without boosts (dash-dotted/blue line) and the NRIA (dashed/green lines). Experimental data as specified in the inserts, see also refs. $[14,16]$.
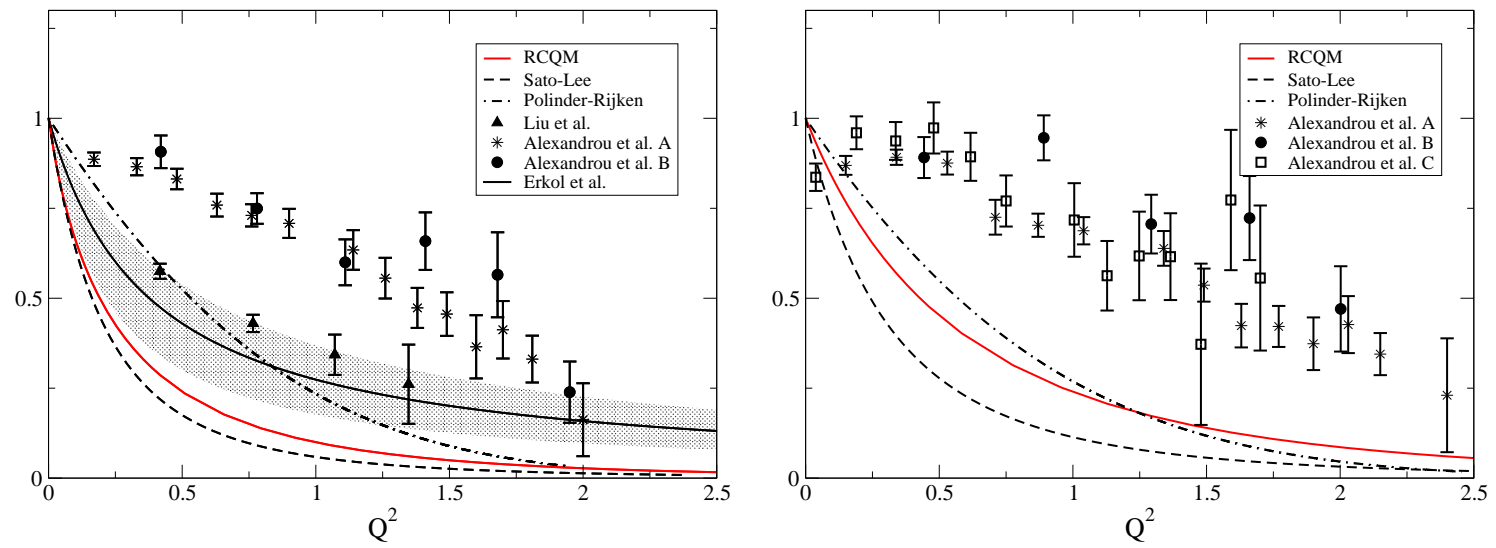

Figure 8: Predictions of the strong vertex form factors $G_{\pi N N}$ (left) and $G_{\pi N \Delta}$ (right) by the psGBE RCQM (solid/red line) in comparison to parametrizations from the dynamical meson-baryon models of Sato-Lee [24] and Polinder-Rijken [25, 26] as well as results from lattice QCD calculations [27, 29, 30, 28] (cf. the legend); in the left panel the shaded area around the result by Erkol et al. (thick solid line) gives their theoretical error band. For more details see ref. [23].

\section{Acknowledgement}

I am grateful to the organizers of the LC2010 conference for having given me the opportunity to present this review of recent achievements within point-form relativistic quantum mechanics at a meeting that primarily focussed on light-front quantum mechanics and light-front field theory.

The results discussed here have arisen over several years with essential contributions by K. Berger, S. Boffi, L. Canton, K.-S. Choi, B. Desplanques, K. Glantschnig, L. Y. Glozman, R. Kainhofer, W. Klink, T. Melde, Z. Papp, M. Radici, B. Sengl, L. Theussl, K. Varga, and R. F. Wagenbrunn. 


\section{References}

[1] L. Y. Glozman and D. O. Riska, Phys. Rep. 268, 263 (1996)

[2] L. Y. Glozman, Z. Papp, and W. Plessas, Phys. Lett. B 381, 311 (1996)

[3] L. Y. Glozman, W. Plessas, K. Varga, and R. F. Wagenbrunn, Phys. Rev. D 58, 094030 (1998)

[4] K. Glantschnig, R. Kainhofer, W. Plessas, B. Sengl, and R. F. Wagenbrunn, Eur. Phys. J. A 23, 507 (2005)

[5] L. Y. Glozman, Z. Papp, W. Plessas, K. Varga, and R. F. Wagenbrunn, Phys. Rev. C 57, 3406 (1998)

[6] L. Theussl, R. F. Wagenbrunn, B. Desplanques, and W. Plessas, Eur. Phys. J. A 12, 91 (2001)

[7] U. Löring, B. C. Metsch, and H. R. Petry, Eur. Phys. J. A 10, 395 (2001); ibid. A 10, 447 (2001)

[8] K. Nakamura et al. [Particle Data Group], J. Phys. G 37, 075021 (2010)

[9] N. Mathur et al. Phys. Lett. B 605, 137 (2005)

[10] T. Melde, W. Plessas, and B. Sengl, Phys. Rev. D 77, 114002 (2008)

[11] T. Melde, L. Canton, W. Plessas, and R. F. Wagenbrunn, Eur. Phys. J. A 25, 97 (2005)

[12] T. Melde, K. Berger, L. Canton, W. Plessas and R. F. Wagenbrunn, Phys. Rev. D 76, 074020 (2007)

[13] R. F. Wagenbrunn, S. Boffi, W. Klink, W. Plessas and M. Radici, Phys. Lett. B 511, 33 (2001)

[14] S. Boffi, L. Y. Glozman, W. Klink, W. Plessas, M. Radici and R. F. Wagenbrunn, Eur. Phys. J. A 14, $17(2002)$

[15] K. Berger, R. F. Wagenbrunn and W. Plessas, Phys. Rev. D 70, 094027 (2004)

[16] L. Y. Glozman, M. Radici, R. F. Wagenbrunn, S. Boffi, W. Klink and W. Plessas, Phys. Lett. B 516, $183(2001)$

[17] K. S. Choi, W. Plessas and R. F. Wagenbrunn, Phys. Rev. D 82, 014007 (2010)

[18] K. S. Choi, W. Plessas and R. F. Wagenbrunn, Phys. Rev. C 81, 028201 (2010)

[19] H. W. Lin and K. Orginos, Phys. Rev. D 79, 034507 (2009)

[20] G. Erkol, M. Oka, and T. T. Takahashi, Phys. Lett. B 686, 36 (2010)

[21] F. J. Jiang and B. C. Tiburzi, Phys. Rev. D 78, 017504 (2008)

[22] F. J. Jiang and B. C. Tiburzi, Phys. Rev. D 80, 077501 (2009)

[23] T. Melde, L. Canton and W. Plessas, Phys. Rev. Lett. 102, 132002 (2009)

[24] T. Sato and T. S. H. Lee, Phys. Rev. C 54, 2660 (1996)

[25] H. Polinder and T. A. Rijken, Phys. Rev. C 72, 065210 (2005)

[26] H. Polinder and T. A. Rijken, Phys. Rev. C 72, 065211 (2005)

[27] C. Alexandrou et al. Phys. Rev. D 76, 094511 (2007)

[28] G. Erkol, M. Oka and T. T. Takahashi, Phys. Rev. D 79, 074509 (2009)

[29] K. F. Liu, S. J. Dong, T. Draper, and W. Wilcox, Phys. Rev. Lett. 74, 2172 (1995)

[30] K. F. Liu et al., Phys. Rev. D 59, 112001 (1999) 